\title{
Anomalous Particle Size Dependence of Magnetic Relaxation Phenomena in Goethite Nanoparticles
}

\author{
Cathrine Frandsen, ${ }^{1, *}$ Daniel Esmarch Madsen, ${ }^{1}$ Chris B. Boothroyd,${ }^{2}$ Steen Mørup ${ }^{1}$
}

1 Department of Physics, Technical University of Denmark, DK-2800 Kgs. Lyngby, Denmark

2 Ernst Ruska-Centrum und Peter Grünberg Institut, Forschungszentrum Jülich, D-52425 Jülich, Germany

* Corresponding author's e-mail address: fraca@fysik.dtu.dk

RECEIVED: October 1, 2015 * REVISED: December 19, 2015 * ACCEPTED: December 19, 2015

THIS PAPER IS DEDICATED TO DR. SVETOZAR MUSIĆ ON THE OCCASION OF HIS 70

Abstract: By use of Mössbauer spectroscopy we have studied the magnetic properties of samples of goethite nanoparticles with different particle size. The spectra are influenced by fluctuations of the magnetization directions, but the size dependence is not in accordance with the Néel-Brown expression for superparamagnetic relaxation of the magnetization vectors of the particles as a whole. The data suggest that the magnetic fluctuations can be explained by fluctuations of the magnetization directions of small interacting grains within the particles.

Keywords: goethite ( $\alpha-\mathrm{FeOOH})$, Mössbauer spectroscopy, magnetic relaxation, nanoparticles.

\section{INTRODUCTION}

G OETHITE $(\alpha-\mathrm{FeOOH})$ is an antiferromagnetic mineral, which usually is found in the form of aggregated nanoparticles. The crystal structure of goethite is orthorhombic with space group Pnma. The magnetic properties of goethite have been studied extensively by Mössbauer spectroscopy, ${ }^{[1-11]}$ magnetization measurements $[9,11,12]$ and neutron scattering. ${ }^{[1,11,13,14]}$ The magnetic properties of nanocrystalline goethite samples often differ from those of non-interacting nanoparticles.

For an isolated particle with uniaxial anisotropy the superparamagnetic relaxation time is given by

$$
\tau=\tau_{0} \exp \left(\frac{K V}{k_{\mathrm{B}} T}\right)
$$

where $\tau_{0}$ is on the order of $10^{-9}-10^{-13} \mathrm{~s}, K$ is the magnetic anisotropy constant, $V$ is the particle volume, $k_{\mathrm{B}}$ is Boltzmann's constant and $T$ is the temperature. In goethite nanoparticles the dependence of magnetic fluctuations on particle volume and the temperature is usually not in accordance with Eq. (1). The reason for the anomalous behavior of goethite can be explained by inter-particle interactions and by magnetic fluctuations in interacting grains in the interior of the particles. ${ }^{[10,11]}$ In this paper, we present a study of three goethite samples with different particle volumes and discuss the size dependence of the magnetic properties.

\section{EXPERIMENTAL}

Three goethite powder samples with different particle sizes, in the following referred to as G1, G2 and G3, were obtained from the company NanoChemonics Inc. A study of the magnetic properties of sample $\mathrm{G} 1$ has been published earlier. ${ }^{[11]}$

X-ray diffraction (XRD) was performed using a Bruker D8 Advance powder diffractometer with a Co anode $(\lambda=$ $1.79 \AA$ A). Rietveld refinement of the orthorhombic Pnma structure of goethite was performed using the WINPOW program, a modified version of the LHMP1 program. ${ }^{[15]}$

Transmission electron microscopy (TEM) images of the samples were taken using an FEI Tecnai T20 G2 microscope with a thermionic $\mathrm{LaB}_{6}$ filament and an accelerating voltage of $200 \mathrm{kV}$.

${ }^{57} \mathrm{Fe}$ Mössbauer spectroscopy was carried out using conventional constant acceleration spectrometers with 
sources of ${ }^{57} \mathrm{Co}$ in rhodium and calibration was carried out using a $12.5 \mu \mathrm{m}$ foil of $\alpha$-Fe at room temperature. Spectra obtained at temperatures down to $20 \mathrm{~K}$ were recorded in a close cycle helium refrigerator from APD Cryogenics.

\section{RESULTS}

Figure 1 shows XRD data for sample G3 and Figure 2 shows TEM images for samples $G 2$ and G3. The XRD and TEM data for sample $\mathrm{G} 1$ have been published earlier ${ }^{[11]}$ and the XRD data from sample $\mathrm{G} 2$ are similar to those of G3. The structural characterization by XRD and TEM is summarized in Table 1. Sample $G 1$ consists of pseudo-spherical $\sim 5 \mathrm{~nm}$ particles (discussed in Ref. 11), while samples $G 2$ and G3 (Figure 2) are dominated by rod-shaped particles. As can be seen from the TEM images in Figures $2 a-f$, the rods in sample $\mathrm{G} 2$ are $~ 3-10 \mathrm{~nm}$ wide and $\sim 40 \mathrm{~nm}$ long. The rods in sample $\mathrm{G} 3$ are slightly thicker (Figures $2 \mathrm{~g}, \mathrm{~h}$ ). Rietveld refinement of XRD data was performed using the orthorhombic Pnma structure of goethite. The refinements were performed with Voigtian peak profiles and the background was modelled with Chebyshev polynomials. The unit cell parameters (Table 1), a Lorentzian profile broadening parameter, an overall temperature factor and background parameters were refined. The instrumental broadening was assumed to be Gaussian and was known from measurement of a corundum $\left(\mathrm{Al}_{2} \mathrm{O}_{3}\right)$ standard, while the sample broadening was assumed to be Lorentzian. Assuming the line broadening is only due to the finite particle size, the crystallite sizes were determined and are shown in Table 1.

Some of the Mössbauer spectra from the samples, obtained at the indicated temperatures, are shown in Figure 3. At the lowest temperatures the spectra are magnetically

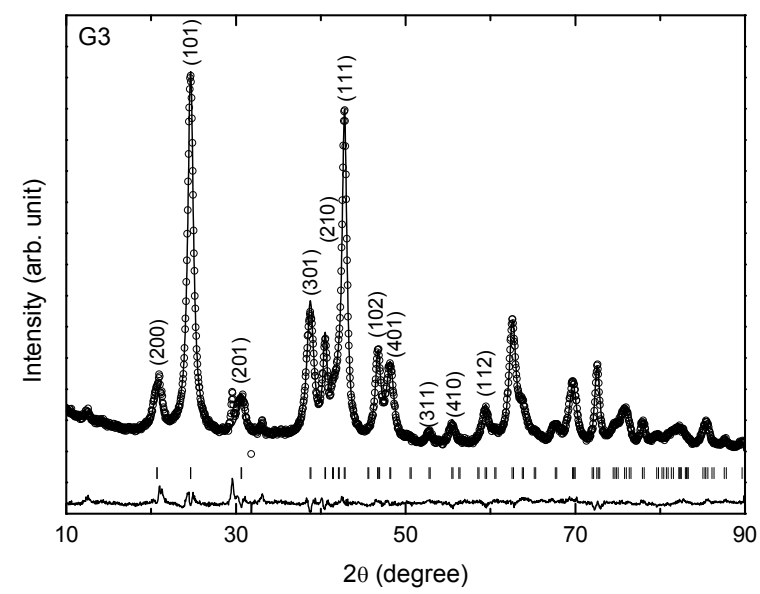

Figure 1. XRD patterns for sample G3. The refined model and difference between the model and the measurements are displayed. The most pronounced reflections up to 60 degrees are indexed.
Table 1. Unit cell parameters, $a, b$, and $c$, and crystalline correlation lengths $d$ along principal axes obtained from the Rietveld refinement of the XRD data. The Volume $V_{X R D}$ is calculated as the product of the crystalline correlation lengths. Particle widths $\mathrm{W}_{\text {TEM }}$ and lengths $\mathrm{L}_{\mathrm{TEM}}$ are measured from TEM images. Average particle volume, $V_{T E M}$, is calculated from the measured particle dimensions (for $\mathrm{G} 1$ see Ref. 11 for details, for G2 and G3 from the average $W_{\text {TEM }}$ squared times $\left.L_{T E M}\right)$. Note $V_{X R D}$ is a volume-weighted measure, while $V_{\text {TEM }}$ is not.

\begin{tabular}{cccc}
\hline & $\mathrm{G} 1$ & $\mathrm{G} 2$ & $\mathrm{G} 3$ \\
\hline$a / n m$ & $0.9980(1)$ & $0.99610(8)$ & $0.99666(8)$ \\
$b / \mathrm{nm}$ & $0.30236(3)$ & $0.30246(2)$ & $0.30251(1)$ \\
$c / n m$ & $0.4604(1)$ & $0.46223(5)$ & $0.46221(5)$ \\
$d_{[100] \times R D} / \mathrm{nm}$ & 3.9 & 7.6 & 7.8 \\
$d_{[010] \times \mathrm{XRD}} / \mathrm{nm}$ & 8.0 & 23.9 & 24.9 \\
$d_{[001] \times \mathrm{RD}} / \mathrm{nm}$ & 4.0 & 6.7 & 9.5 \\
$\mathrm{~V}_{\text {XRD }} / \mathrm{nm}^{3}$ & 127 & 1222 & 1829 \\
$\mathrm{~W}_{\text {TEM }} / \mathrm{nm}$ & $\sim 3-6$ & $\sim 3-10$ & $\sim 5-12$ \\
$\mathrm{~L}_{\text {TEM }} / \mathrm{nm}$ & $\sim 3-6$ & $\sim 40$ & $\sim 40$ \\
$\mathrm{~V}_{\text {TEM }} / \mathrm{nm}^{3}$ & $\sim 100$ (Ref. 11) & $\sim 2250$ & $\sim 2900$ \\
\hline
\end{tabular}

split with magnetic hyperfine fields around 49.5 $\mathrm{T}$, isomer shifts of $0.49 \mathrm{~mm} \mathrm{~s}^{-1}$ and quadrupole shifts of $-0.13 \mathrm{~mm} \mathrm{~s}^{-1}$. There is no indication of impurity phases. At higher temperatures, the spectra of all the samples show an asymmetric line broadening, indicating that the magnetic properties are influenced by magnetic fluctuations, as typically seen in goethite. ${ }^{[10,11]}$ At temperatures above $\sim 220 \mathrm{~K}$ the spectra from $\mathrm{G} 1$ consist of a doublet, suggesting that all particles in this sample exhibit fast superparamagnetic relaxation. The spectra from $\mathrm{G} 2$ and $\mathrm{G} 3$ consist of a superposition of broadened sextets and doublets at $300 \mathrm{~K}$.

\section{DISCUSSION}

In goethite, the magnetic anisotropy constant, $K$, is approximately $10^{5} \mathrm{Jm}^{-3}$ (Ref. 11). In sample $\mathrm{G1}$, the average particle volume is about $100 \mathrm{~nm}^{3}$ (determined in Ref. 11, from XRD and TEM analysis). Assuming that $\tau_{0}$ is on the order of $10^{-11} \mathrm{~s}$ we find from Eq. (1) that $\tau \approx 10^{-10} \mathrm{~s}$ at $300 \mathrm{~K}$ and $\approx 1.4 \times 10^{-8} \mathrm{~s}$ at $100 \mathrm{~K}$. This is at least qualitatively in accordance with the evolution of the Mössbauer spectra of $G 1$, shown in Figure 3. Using the volumes determined by XRD and TEM (Table 1) for the rods of samples G2 and G3, we find from Eq. (1) that $\tau$ is on the order of a second or longer at room temperature, even if we use the smallest volume (around $1200 \mathrm{~nm}^{3}$ ) and if we assume a value for $\tau_{0}$ as small as $10^{-13} \mathrm{~s}$. With such long relaxation times the spectra 

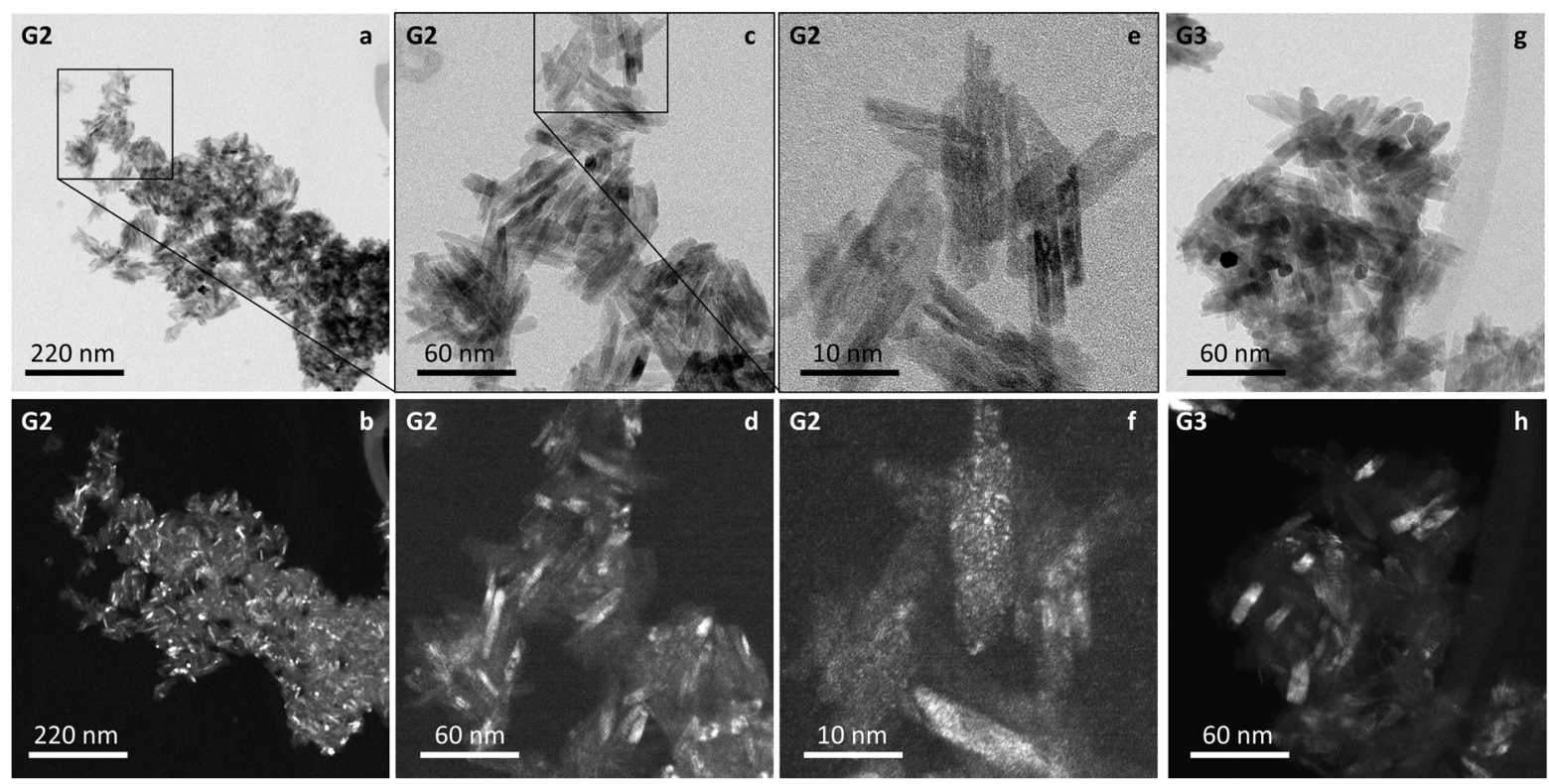

Figure 2. TEM images of samples $G 2$ and $G 3 ; a, c$, e and $g$ are bright field images, $b, d, f$ and $h$ are the corresponding dark field images.

should consist of sextets with sharp lines. Therefore, the presence of doublets and sextets with broadened lines at $300 \mathrm{~K}$ cannot be due to superparamagnetic relaxation of the magnetization directions of the particles as a whole.

Recent Mössbauer studies of different goethite nanoparticles ${ }^{[10,11]}$ have shown that the evolution of the spectra as a function of temperature can be described by a model for interacting particles or grains. The TEM images in Figure 2 show indeed that the particles form agglomerates

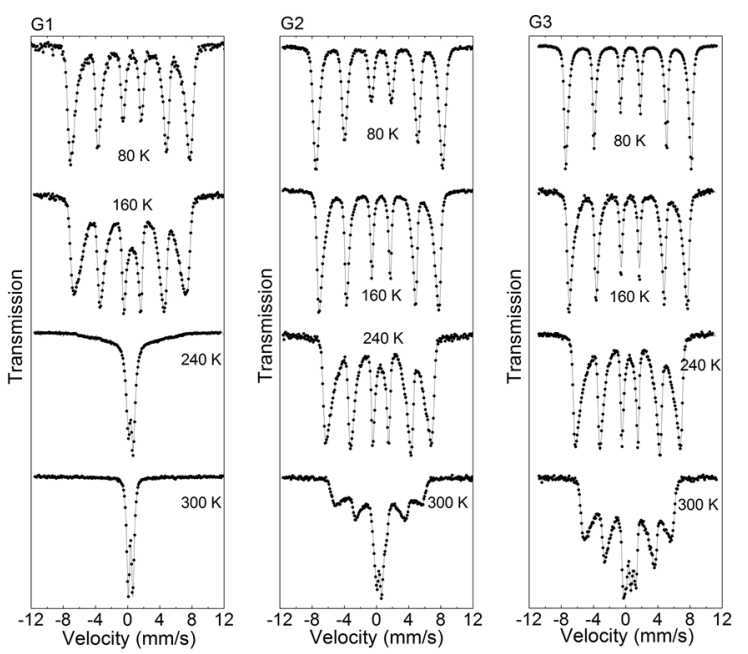

Figure 3. Mössbauer spectra of samples G1, G2 and G3. The spectra were obtained at the indicated temperatures. Fits to data are shown. with a tendency for alignment of the particles. High-resolution TEM images of goethite particles have also shown that the particles often contain many defects in the form of lowangle grain boundaries, such that each particle can be described as consisting of a number of interacting grains with nearly oriented attachment. ${ }^{[10]}$ This sub-particle structure is also supported by the bright and dark field images in Figure 2 , where the diffracting regions (Figures $2 d, h$ ) often are smaller than the rod-sizes (Figures $2 \mathrm{c}, \mathrm{g}$ ), and where diffracting regions down to grain sizes of a few nanometers are seen (Figure $2 \mathrm{f}$ ).

The magnetic energy of interacting goethite nanoparticles or grains may be written ${ }^{[10,11]}$

$$
E(\theta) \approx K V \sin ^{2} \theta-J_{\text {eff }} M_{0}^{2}(T) b(T) \cos \theta
$$

where $K$ is the magnetic anisotropy constant, $V$ is the particle or grain volume, $J_{\text {eff }}$ is an effective exchange interaction constant describing the magnetic interaction between particles or grains, $M_{0}(T)$ is the sublattice magnetization and

$$
b(T)=\frac{|\langle\vec{M}(T)\rangle|}{M_{0}(T)}
$$

is the order parameter. In thermal equilibrium, the order parameter can be calculated by the use of Boltzmann statistics: 


$$
b(T)=\frac{\int_{0}^{\pi} \exp \left(-\frac{E(\theta)}{k_{\mathrm{B}} T}\right) \sin \theta \cos \theta d \theta}{\int_{0}^{\pi} \exp \left(-\frac{E(\theta)}{k_{\mathrm{B}} T}\right) \sin \theta d \theta}
$$

The temperature dependence of the order parameter $b(T)$ can be found by solving numerically Eqs. (2) and (4). If the magnetic fluctuations are fast, the magnetic hyperfine field is proportional to $b(T) \cdot[10,11,16,17]$

In samples of nanoparticles there is inevitably a distribution of anisotropy energies and effective exchange interaction constants. Therefore, there will be a distribution of order parameters and thus magnetic hyperfine fields at finite temperatures. In the distributions of magnetic hyperfine fields, obtained from the superferromagnetism model, $[4,16,17]$ the values of the anisotropy energy, $K V$ and the interaction energy parameter $T_{p}^{0}$ are free parameters for each quantile, where the quantile, $f$, is defined as ${ }^{[11,16]}$

$$
f=\int_{0}^{B_{f}(T)} p\left(B_{h f}(T)\right) d B_{h f} .
$$

$T_{p}^{0}$ is defined as the ordering temperature for a sample with zero anisotropy $(K V=0)$ and is given by the expres$\operatorname{sion}^{[4,16,17]}$

$$
T_{p}^{0}=\frac{J_{\text {eff }} M_{0}\left(T_{p}^{0}\right)^{2}}{3 k_{\mathrm{B}}}
$$

The interaction energy, $E_{i}(T)=J_{\text {eff }} M_{0}(T)^{2} b(T)$ depends on temperature, because both $b(T)$ and $M_{0}^{2}(T)$ are temperature dependent. At low temperatures the interaction energy is approximately given by $E_{\mathrm{int}}=3 k_{\mathrm{B}} T_{p}^{0}$.

Figure 4 shows the estimated values of $K V / k_{\mathrm{B}}$ and $E_{\text {int }} / k_{B}$ as a function of the quantile for the three samples, obtained from the superferromagnetism model. The values of $K V / k_{\mathrm{B}}$ and $E_{\text {int }} / k_{B}$ vary as a function of the quantile. In order to make a comparison of the three samples, we have estimated the values around the $50 \%$ quantile. They are given in Table 2. The last column shows calculated values of $K V_{\text {TEM }} / k_{\mathrm{B}}$ with $K=10^{5} \mathrm{~J} \mathrm{~m}^{-3}$. For sample $\mathrm{G} 1 \mathrm{KV} / \mathrm{k}_{\mathrm{B}}$ and $K V_{\text {TEM }} / k_{\mathrm{B}}$ have the same order of magnitude, indicating that the relaxation phenomena may be dominated by fluctuations of the magnetization vectors of the particles as a whole. For samples $\mathrm{G} 2$ and $\mathrm{G} 3$ the values of $K V_{\mathrm{TEM}} / k_{\mathrm{B}}$ are larger than the values of $K V / k_{\mathrm{B}}$ by more than an order of magnitude. This again emphasizes that the magnetic fluctuations in these samples cannot be fluctuations of the magnetization vectors of the particles as a whole, but rather can be described by fluctuations of the magnetization vectors of smaller grains.

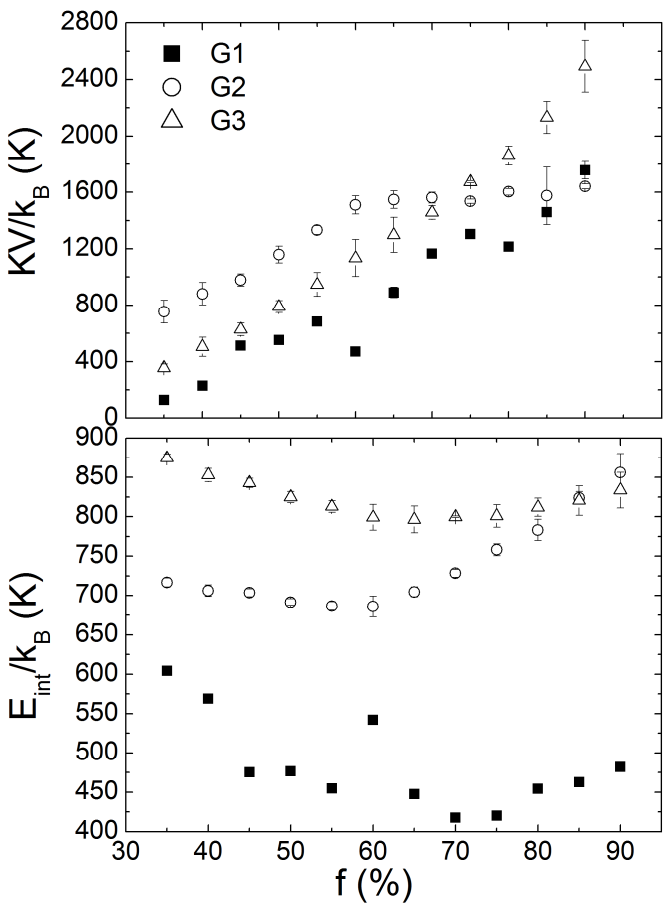

Figure 4. The values of the parameters $K V / k_{\mathrm{B}}$ and $E_{\text {int }} / k_{\mathrm{B}}$ as a function of the quantile, $f$ for samples $G 1, G 2$ and $G 3$.

Table 2. Approximate values of the anisotropy energies and interaction energies around the 50\% quantile for the three samples. The last column shows calculated values of $K V_{\text {TEM }} / k_{B}$.

\begin{tabular}{cccc}
\hline Sample & $K V / K_{\mathrm{B}}(\mathrm{K})$ & $E_{\text {int }} / K_{\mathrm{B}}(\mathrm{K})$ & $K V_{\mathrm{TEM}} / \mathrm{KB}_{\mathrm{B}}(\mathrm{K})$ \\
\hline $\mathrm{G} 1$ & 550 & 470 & 725 \\
$\mathrm{G} 2$ & 750 & 700 & 16300 \\
$\mathrm{G} 3$ & 1200 & 825 & 21000 \\
\hline
\end{tabular}

\section{CONCLUSIONS}

Magnetic relaxation in samples of goethite nanoparticles with different average particle sizes has been studied by use of Mössbauer spectroscopy. The size dependence of the relaxation phenomena is not in accordance with the Néel-Brown expression for the superparamagnetic relaxation time for relaxation of the magnetization of the particles as a whole. The results rather suggest that the relaxation is dominated by magnetic fluctuations of the magnetization of much smaller, interacting grains.

Acknowledgment. We thank A. A. Berhe for help with the samples. We acknowledge funding from the Danish Council for Independent Research. 


\section{REFERENCES}

[1] J. B. Forsyth, I. G. Hadley, C. E. Johnson, J. Phys. C 1968, 1, 179.

[2] A. M. van der Kraan, J. J. van Loef, Phys. Lett. 1966, 20, 614 .

[3] T. Shinjo, J. Phys. Soc. Japan 1966, 21, 917.

[4] S. Mørup, M. B. Madsen, J. Franck, J. Villadsen, C. J. W. Koch, J. Magn. Magn. Mater. 1983, 40, 163.

[5] C. J. W. Koch, M. B. Madsen, S. Mørup, Surf. Sci. 1985, 156, 249.

[6] E. Murad, Amer. Miner. 1982, 67, 1007.

[7] S. Bocquet, R. J. Pollard, J. D. Cashion, Phys. Rev. B 1992, 46, 11657.

[8] C. A. Barrero, J. D. Betancur, J. M. Greneche, G. F. Goya, T. S. Berquó, Geophys. J. Int. 2006, 164, 331.

[9] Q. A. Pankhurst, L. F. Barquín, J. S. Lord, A. Amato, U. Zimmermann, Phys. Rev. B 2012, 85, 174437.

[10] D. E. Madsen, L. Cervera-Gontard, T. Kasama, R. E.
Dunin-Borkowski, C. B. Koch, M. F. Hansen, C. Frandsen, S. Mørup, J. Phys.: Condens. Matter 2009, 21, 016007.

[11] E. Brok, C. Frandsen, D. E. Madsen, H. Jacobsen, J. O. Birk, K. Lefmann, J. Bendix, K. S. Pedersen, C. B. Boothroyd, A. A. Bethe, G. G. Simeoni, S. Mørup, J. Phys. D: Appl. Phys. 2014, 47, 365003.

[12] F. Martin-Hernandez, M. M. Garcia-Hernández, Geophys. J. Int. 2010, 181, 756.

[13] S. Bocquet, S. J. Kennedy, J. Magn. Magn. Mater. 1992, 109, 260.

[14] J. M. D. Coey, A. Barry, J.-M. Brotto, H. Rakoto, S. Brennan, W. N. Mussel, A. Collomb, D. Fruchard, J. Phys.: Condens. Matter 1995, 7, 759.

[15] C. J. Howard, R J. Hill, AAEC (now ANSTO) Report M112, Lucas Heights Research Laboratory, 1986.

[16] M. F. Hansen, C. Bender Koch, S. Mørup, Phys. Rev. B 2000, 62, 1124.

[17] S. Mørup, C. Frandsen, and M. F. Hansen, Beilstein J. Nanotechnol. 2010, 1, 48. 